\title{
Rate of cognitive decline in Alzheimer's disease stratified by age
}

Running title - Rate of cognitive decline in Alzheimer's disease stratified by age

\section{Authors}

Karen Stanley ${ }^{1,2}$

Tim Whitfield ${ }^{1,2}$

Karoline Kuchenbaecker ${ }^{2}$

Oliver Sanders ${ }^{2}$

Tim Stevens ${ }^{2}$

Zuzana Walker ${ }^{1,2}$

$1=$ Essex Partnership University Foundation Trust, St Margaret's Hospital, The Plain, Epping, Essex, CM16 6TN

$2=$ UCL Division of Psychiatry, $6^{\text {th }}$ Floor Maple House, 149 Tottenham Court Road, London, W1T 7NF

The author Karen Stanley is the corresponding author, correspondence email and address: kstanley@doctors.net.uk; St Margaret's Hospital, The Plain, Epping, Essex, CM16 6TN; United Kingdom. 


\section{Abstract}

Background: There is only limited information available about the effect of age on course of cognitive decline in patients with onset of Alzheimer's Disease (AD) over the age of 64 years.

Objective: We compared the rate of, and factors affecting, cognitive decline in patients with $A D$ aged $<65$ years (young-onset $A D$ ), 65-74 years (middle-onset $A D$ ), and $\geq 75$ years (late-onset $A D)$.

Method: The study used longitudinal data from the Essex Memory Clinic which included a total of 305 participants; 56 had YOAD, 73 had MOAD, and 176 had LOAD. The rate of cognitive decline was measured using scores from the Mini Mental State Examination (MMSE), and the data were examined using multilevel models analysis.

Results: There was evidence of a difference in cognitive decline across the age groups with the YOAD group declining 2.8 MMSE points per year, those with MOAD declined 2.0 MMSE points per year and the LOAD group declined 1.4 MMSE points per year.

Conclusions: Patients with LOAD have a better prognosis than YOAD and MOAD. However, even between the MOAD and LOAD groups, age is a significant predictor of cognitive decline, with older patients having a more benign course.

Declaration of interests: none

Keywords: Alzheimer's disease; rate of decline; cognitive decline; young-onset; dementia 


\section{Introduction}

Alzheimer's disease $(A D)$ is conventionally defined as young- and late-onset $A D$ (YOAD, LOAD) equating to age at onset $<65$ years and $\geq 65$ years respectively. There is an abundance of literature looking at the relationship between age, both as a continuum and dichotomized, with the progression of cognitive impairment in AD. The majority of studies found a faster rate of cognitive decline in younger patients when looking either at age at which a diagnosis of $A D$ is ascertained [1-12], or the age of onset, that is when symptoms first begin [13-14]. Some found no association between age of onset or diagnosis and rate of decline [15-20]. However, few studies have examined the effect of age on rate of decline within the LOAD group. As the prevalence of $A D$ is increasing, and the UK population continues to age with a large AD cohort now aged over 65 years [21] there is a greater need for an understanding of the speed of decline of the disease and how age may affect this.

In this study we looked at the age at which a diagnosis of AD was given and the rate of cognitive decline. Age at diagnosis is considered to be more reliable than age at onset which is dependent on patients/family members' historical accounts and therefore subject to bias. The age at which a diagnosis of $A D$ was given was split into $<65$ years and $>65$ years in line with the diagnostic classification of AD into YOAD and LOAD respectively. We further stratified the over $65 \mathrm{~s}$ in order to assess whether there was an effect of age on decline even within the over $65 \mathrm{~s}$. The LOAD group was subdivided into $65-74$ years and $\geq 75$ years. The $65-74$ years old age group were defined as middle-onset $A D(M O A D)$ and the $\geq 75$ years old group were 
defined as late-onset AD (LOAD). The aim was to compare the rate of cognitive decline in patients with YOAD, MOAD and LOAD. We also sought to identify any additional factors that may influence the rate of decline including sex, years in education and vascular risk factors.

\section{Method}

\section{Participants}

Patients were identified through the Essex Memory Clinic (EMC), a specialised memory assessment service that provides a detailed assessment of patients presenting with cognitive problems over the age of 40 following referrals from General Practitioners (GP) and allied health professionals. The results of the assessments are stored on the memory clinic's database following patient consent. If the outcome of an assessment reveals a diagnosis of dementia then patients are usually discharged from the EMC and subsequently followed up and reviewed locally by their respective Older Adult Mental Health team. Patients were given a consensus diagnosis following discussion between 2 Consultant Old Age Psychiatrists, a Clinical Psychologist and a Memory Clinic Nurse. Between March 1993 and July 2015, 1827 patients were assessed (see figure 1). Of those, 612 had a diagnosis of $A D$ at their most recent clinic visit. The inclusion criteria were patients aged 40 years or greater; meeting diagnostic criteria for possible or probable AD at their most recent visit (22); and at least 12 months follow up with repeated MMSE score (23) following the diagnosis of dementia. Patients were excluded if they had history of severe mental illness or substance misuse, or a change of diagnosis following 
discharge. Three hundred and five patients were included in the final analysis (YOAD $n=56 ;$ MOAD $n=73 ;$ LOAD $n=176)$.

Figure 1: study flowchart

\section{Procedure}

For each eligible patient demographic data, smoking (current and ex combined into one value versus never smoked), drinking habits, and vascular risk factors including history of myocardial infarction, hypertension, hypercholesteraemia, diabetes, and the Hachinski Ischaemic Score (HIS) were recorded [24]. We considered baseline MMSE score to be the score obtained at the time of AD diagnosis. Follow-up MMSE scores were taken at subsequent routine clinical assessments. The data were then transferred into SPSS (version 19) for analysis.

\section{Statistical analysis}

For baseline group comparisons, Mann-Whitney, $\mathrm{X}^{2}$ tests, and independent $\mathrm{t}$ tests were used where appropriate depending on the variable and distribution type. In view of the hierarchical structure of our longitudinal data i.e. repeated measures of MMSE scores for each individual, a multilevel models analysis approach was adopted. This approach improves statistical power, as it accounts for within-person correlations over time, allows different numbers of assessments, can be used with datasets with missing data, and allows for varying time intervals between assessments [25]. 
Multilevel models were used to assess associations between age at diagnosis and the rate of cognitive decline as measured by the MMSE. All assessments, including baseline, were taken into account. A random intercept and random slope were used for the analysis. The intercept represented the initial MMSE score, and the slope represented the rate of change in MMSE scores across time. The first model included terms for age at diagnosis; time; sex; years of education and the interactions between age at diagnosis and time; sex and time; and years of education and time, with the MMSE score as the dependent variable. Any nonsignificant covariates were then removed from the model, and the variables smoking, alcohol use, and vascular risk factors were added to see if there was an association between them and initial MMSE status and/or rate of decline. In the final model reported here, only variables with a significant association with initial MMSE status and/or rate of decline were retained. From this model, the baseline MMSE and annual change in MMSE for patients with different age at diagnosis could be calculated.

The continuous variable education was centred (mean subtracted from individuals' scores) before the analysis. Age was trichotomized ( $<65$ years, 65 to 74 years and $>74$ years). All analyses were conducted using SPSS version 21 , with the significance level set at .05 .

\section{Ethics}

The relevant Local Research Ethics Committee (NRES Committee South West Cornwall and Plymouth and Exeter) gave ethical approval for the study. 


\section{$\underline{\text { Results }}$}

\section{Study Demographics}

Fifty six (18\%) patients had YOAD, 73 (24\%) had MOAD, and $176(58 \%)$ had LOAD (see Tables 1 and 2). The mean age at diagnosis of persons with YOAD was 59.7 years (range 48 to 64 ) and of MOAD 71.1 years (range 65 to 74 ). LOAD comprised a larger sample size of 176, mean age 80.2 years (range 75 to 92). There was no evidence of a difference in sex distribution between the three groups. Those with YOAD had an increased number of years in education compared to those with MOAD and LOAD $(p<.01)$. There was no evidence of a difference between the duration of symptoms prior to diagnosis in the three groups. However, the first symptom of YOAD patients was more likely to be changes in mood such as anxiety and/or depression. There was also no evidence of a difference to our findings after adjusting for length of symptoms. The baseline severity (as measured using the MMSE and CAMCOG) of patients with YOAD was worse compared to those with MOAD and LOAD $(p<.001)$. There was no difference in baseline severity between the MOAD and LOAD groups. The MOAD group had a greater number of follow ups compared to YOAD $(p<.01)$. Of the possible covariates affecting cognitive decline the MOAD and LOAD groups had a higher rate of hypertension $(p<.01)$; there were no differences in the other vascular measures, or smoking and alcohol habits. Fewer individuals with LOAD were taking the anti-dementia drug memantine in addition to their cholinesterase inhibitor $(p<.01)$.

Table 1 Baseline demographics and clinical characteristics between individuals with YOAD (<65 yrs), MOAD (65-74 yrs) and LOAD ( $>75 \mathrm{yrs)}$ 
Table 2 Clinical characteristics (percent yes)

\section{Findings from the analysis}

The initial model included terms for age at diagnosis; time; sex; years of education and the interactions between age at diagnosis and time; sex and time; and years of education and time, with the MMSE score as the dependent variable. All covariates were significant at $p<.05$ with the exception of the interactions between time and years of education, and time and sex. The non-significant terms were dropped from the model and the following parameter estimates were obtained: for a patient with YOAD of average education and female sex, the initial MMSE status was 19.4 points. At baseline the MOAD group had an MMSE score 4.8 points higher $(p<.01)$; individuals with LOAD had an MMSE score that was 3.9 points higher $(p<.01$ versus YOAD); male sex was associated with an increase of 1.1 MMSE points $(p<.05)$; and each extra year of education was associated with an increase of 0.3 MMSE points $(p<.05)$. Regarding rate of change, YOAD patients declined by 0.23 MMSE points per month. Those with MOAD declined by 0.17 MMSE points per month $(p<.05)$, and those with LOAD declined by 0.11 MMSE points per month $(p<.01)$. Using estimated regression lines of MMSE score by time, the rate of decline in patients with YOAD was 2.7 MMSE points per year. At 48 months (in a prototypical YOAD patient with an initial MMSE of 19.4) the estimated MMSE score is 8.5. Patients with MOAD declined 2.0 points per year. At 48 months (in a prototypical MOAD patient with an 
initial MMSE of 24.3) the estimated MMSE score is 16.3. Patients with LOAD declined 1.3 points per year. At 48 months (in a prototypical LOAD patient with an initial MMSE of 23.4) the estimated MMSE score is 18.1 .

To the initial model, the variables smoking, alcohol use, and vascular risk factors were added to see if there was an association between them and initial MMSE status and/or rate of decline. Only diabetic status was significantly associated with initial MMSE status and no variables were associated with rate of decline. In the final model, all non-significant covariates were dropped. In the final model, the initial MMSE score of diabetic patients was 1.7 points higher $(p<.05)$ than non-diabetic patients. All other parameter estimates were essentially unchanged from the initial model, including no change to the level of statistical significance.

To supplement the analyses above, we directly compared initial MMSE status and rates of decline based on the final model for MOAD versus LOAD. In this model, the following parameter estimates were obtained: for a patient with MOAD of average education, female sex, and non-diabetic status the initial MMSE status was 24.0 points. At baseline the LOAD group had an MMSE score 0.8 points lower $(p=.14)$. Regarding rate of change, MOAD patients declined by 0.17 MMSE points per month. Those with LOAD declined by 0.11 MMSE points per month ( $p<.01$; see Figure 2$)$.

Of the 305 cases studied, 54 only had 2 assessment time points. The results of patients with only 2 time points could be perceived as being less reliable. We therefore conducted a sensitivity analysis excluding cases with only 2 scores. The repeated analyses gave broadly similar results. However, additional years of 
education and diabetes were no longer associated with higher initial MMSE status. The rate of decline of early-onset cases decreased (from - 0.23 to -0.20 MMSE points per month). This was still significantly different from zero. This slight reduction in rate of decline meant that there was no longer a significant difference in rate of decline between early- and middle-onset cases. However, there continued to be significant differences in rate of decline between early- and late-onset cases, and middle-onset and late-onset cases.

Figure 2. Initial MMSE status (top) and rates of decline (bottom) for YOAD, MOAD and LOAD.

\section{Discussion}

\section{Summary of main findings}

Patients with YOAD had a baseline MMSE score of 19.3. Patients with MOAD had a higher baseline MMSE score of 24.0, whereas those with LOAD had a score of 23.2 It therefore did not follow a consistent trend that older patients presented with higher MMSE scores at baseline.

In terms of rate of decline from the time of diagnosis individuals with YOAD declined 0.23 MMSE points per month, those with MOAD declined 0.17 MMSE points per month, and those with LOAD declined 0.11 MMSE points per month. This equates to the YOAD individuals declining by 2.7 MMSE points per year, those aged 65 to 74 
declining by 2.0 points per year, and individuals with AD aged 75 and over declining by 1.3 points per year.

The difference in baseline severity between those with YOAD and MOAD/LOAD may have accounted for a faster rate of decline in the YOAD group. Arguing against this, baseline severity was similar between those with MOAD and LOAD and yet the MOAD group declined faster than the LOAD group.

\section{Findings in the context of other literature}

The baseline MMSE for those with YOAD was significantly lower and the rate of decline faster compared to those with MOAD and LOAD. Accurate diagnosis of YOAD is challenging, particularly in the early stages, and as a consequence these patients tend to present late to memory services. Accordingly, they are more cognitively impaired at time of diagnosis, and as a result start antidementia therapy later than individuals with MOAD/LOAD. Yet interestingly in this sample patients with YOAD did not have a longer length of symptoms compared to those with LOAD.

It could be argued that worse baseline severity of patients with YOAD accounts for their faster decline in the present sample. A systematic review looking at predictors of rate of decline concluded that more impaired patients declined faster, although the authors also acknowledged considerable heterogeneity amongst findings [26].

Yet interestingly in our study, whilst patients with YOAD had lower baseline MMSE scores and a faster rate of cognitive decline than those aged $>65$ years, there was a different pattern within the $>65$ years age group. The initial MMSE score of patients 
with MOAD did not significantly differ from that of patients with LOAD, but the MOAD patients declined more quickly. Therefore, whilst greater baseline severity may have contributed to increased rate of decline in the YOAD groups, this cannot be said for the differences in progression between MOAD and LOAD. This suggests that even within patients aged over 65 at diagnosis, age at diagnosis is a significant predictor of decline. Furthermore, in a meta-analysis of $10 \mathrm{AD}$ clinical trials there was no difference in baseline MMSE across any of the age at diagnosis groups (range 48 to 105 years), and yet the older patients declined more slowly. Therefore, in wider research the overall association between age at diagnosis and rate of decline appears to hold across all age groups, regardless of baseline severity [27].

The different rates of decline in AD depending on age at diagnosis may be connected to the observation that age-associated neuropathologies are not mutually exclusive. It is likely that there are interactions of different pathological processes or proteins that seem to aggravate each other [28]. Neuropathological studies suggest older adults are more likely to have simultaneous presence of multiple pathologies in the brain which may accelerate disease progression [28-30]. However from our sample of older patients and similar to other studies [2, 27], we found older adults had a slower rate of decline. A possible explanation for this is that younger individuals have a more "pure" and greater degree of AD pathology, whereas the "older" brain often has mixed neuropathologies [29]. Younger onset of AD is associated with greater grey matter atrophy, increased glucose hypometabolism and greater tau deposition measured using neuroimaging [31]; neuropathologically more severe senile plaques, neurofibrillary tangles and synapse loss [8]; as well as greater deficits in the neurochemical acetylcholine and other neurotransmitters such as 
adrenaline [32]. One large neuropathological study found that, while AD and other neurodegenerative pathologies were associated with progressive cognitive decline, some types of vascular pathology did impair cognition but the effect was stable over time [33]. In our study the MOAD and LOAD groups had a higher rate of hypertension compared to the YOAD group, and there was a difference in the HIS score between the LOAD and YOAD groups suggesting the possible addition of vascular pathology in older patients. In this study, therefore it is likely that the typical LOAD patient had more cerebrovascular disease (CVD) than YOAD patients. However the effects of mixed pathologies on clinical progression in AD remains an area of uncertainty, and there is a need for further studies to combine clinical and neuropathological data in particular to ascertain which pathologies are contributing to cognitive baselines, decline and trajectories [30].

\section{Methodological considerations}

The study included a large sample of patients with AD, and there was a higher rate of inclusion of patients with YOAD compared to similar studies. There was also a substantial length of follow-up for all and a clear effect of age at diagnosis on the rate of cognitive decline was shown. Multilevel modelling is statistically powerful as it allows for repeated measurement of outcomes (MMSE) which can vary in number and interval between patients. All observations, including baseline, were a part of our analysis, but in view of the longitudinal nature of the study only patients with at least 12 months follow up with repeated MMSE score were included. 
The limitations to this study are that there were no post-mortem data available, nor biomarkers to add weight to the clinical diagnosis, and therefore some patients may have had an alternative diagnosis to AD. There are also possible confounding factors which have not been accounted for including APOE genotype, concomitant medications and effectiveness of cholinesterase inhibitor medications such as agent and dose. Additionally, our longitudinal measure of cognition, the MMSE, has limitations. A previous study found that the measure of the rate of changes in MMSE scores were not as reliable when observations were separated by less than 36 months [34]. Furthermore, our data by nature reflects patients who returned to services for follow-up assessment, and therefore may not generalize to patients who are seen only once in clinic. However all patients included in the analysis were assessed in a uniform manner against stringent diagnostic criteria.

A further limitation to consider is using 'age at diagnosis' of AD as opposed to 'age at onset'. This may have introduced bias into the results as some patients may present later to memory services. Also, as discussed earlier, the diagnosis of YOAD in the early stages can be challenging which may result in these patients presenting later to memory services. Subsequently, they are more cognitively impaired at the time of diagnosis, and MMSE rate of change tends to accelerate for people with lower scores. It is therefore possible that age and the severity of cognitive impairment at diagnosis both influence the rate of decline in AD.

\section{Clinical and research implications}

It is important for clinicians to have an understanding of demographic variables affecting the course of cognitive decline in those with $A D$ so that they can relay to 
patients and their carers the likely progression and help them to plan and prepare for the future. In the research setting, when recruiting patients it is important to consider the age at onset of participants, since our and other studies have shown there is a reliable association between age at onset and rate of decline. Researchers testing interventions should consider the use of stratified randomisation based on age at diagnosis rather than age at study entry.

\section{Conclusion}

In conclusion, we found there was some evidence of slower decline at older ages. The greater baseline severity of the YOAD is unlikely to have fully accounted for the increased rate of decline versus those $>65$. The MOAD group declined faster than the LOAD group despite the two groups having similar baseline MMSE scores. Therefore even within patients aged over 65 at diagnosis, age is a significant predictor of decline. Other than age there were no independent variables in our data analysis significantly associated with the rate of decline in AD.

\section{Acknowledgement}

This research did not receive any specific grant from funding agencies in the public, commercial, or not-for-profit sectors.

\section{Declaration of interest}

The authors have no conflict of interest to report.

\section{References}


[1] Bernick C, Cummings J, Raman R, Sun X, Aisen P (2012) Age and rate of cognitive decline in Alzheimer disease: implications for clinical trials. Arch Neuro/69, $901-5$.

[2] Holland D, Desikan RS, Dale AM, McEvoy LK (2012) Alzheimer's Disease Neuroimaging I. Rates of decline in Alzheimer disease decrease with age. PLoS One 7,e42325.

[3] Wilson RS, Beckett LA, Bennett DA, Albert MS, Evans DA (1999) Change in cognitive function in older persons from a community population: relation to age and Alzheimer disease. Arch Neurol 56, 1274-9

[4] Koss E, Edland S, Fillenbaum G, Mohs R, Clark C, Galasko D, Morris JC (1996) Clinical and neuropsychological differences between patients with earlier and later onset of Alzheimer's disease: A CERAD analysis, Part XII. Neurology 46,13641.

[5] Lucca U, Comelli M, Tettamanti M, Tiraboschi P, Spagnoli A (1993) Rate of progression and prognostic factors in Alzheimer's disease: a prospective study. $\mathrm{J} A m$ Geriatr Soc 41,45-9.

[6] Mungas D, Reed BR, Ellis WG, Jagust WJ (2001) The effects of age on rate of progression of Alzheimer disease and dementia with associated cerebrovascular disease. Arch Neurol 58,1243-7.

[7] Panegyres PK, Chen HY (2013) Differences between early and late onset Alzheimer's disease. Am J Neurodegener Dis 2, 300-6.

[8] Sakai M, Hanyu H, Kume K, Sato T, Hirao K, Kanetaka H, Abe S, Kanaya K, Sakurai H, Iwamoto T (2013) Rate of progression of Alzheimer's disease in younger versus older patients: a longitudinal single photon emission computed tomography study. Geriatr Gerontol Int 13,555-62. 
[9] Seltzer B, Sherwin I (1983) A comparison of clinical features in early- and late-onset primary degenerative dementia. One entity or two? Arch Neurol 40,143-6. [10] Wilson RS, Li Y, Aggarwal NT, Barnes LL, McCann JJ, Gilley DW, Evans DA (2004) Education and the course of cognitive decline in Alzheimer disease. Neurology 63, 1198-202.

[11] Wattmo C, Wallin AK (2017) Early- versus late-onset Alzheimer's disease in clinical practice: cognitive and global outcomes over 3 years. Alzheimers Res Ther $\mathbf{9}$, 70.

[12] Wilson RS, Beckett LA, Barnes LL, Schneider JA, Bach J, Evans DA, Bennett DA (2002) Individual differences in rates of change in cognitive abilities of older persons. Psychol Aging 17,179-93.

[13] Jacobs D, Sano M, Marder K, Bell K, Bylsma F, Lafleche G, Albert M, Brandt J, Stern Y (1994). Age at onset of Alzheimer's disease: relation to pattern of cognitive dysfunction and rate of decline. Neurology 44,1215-20.

[14] van der Vlies AE, Koedam EL, Pijnenburg YA, Twisk JW, Scheltens P, van der Flier WM (2009) Most rapid cognitive decline in APOE epsilon4 negative Alzheimer's disease with early onset. Psychol Med 39, 1907-11.

[15] Bowler JV, Munoz DG, Merskey H, Hachinski V (1998) Factors affecting the age of onset and rate of progression of Alzheimer's disease. J Neurol Neurosurg Psychiatry 65,184-90.

[16] Bracco L, Gallato R, Grigoletto F, Lippi A, Lepore V, Bino G, Lazzaro M, Carella F, Piccolo T, Pozzilli C, Giometto B, Amaducci L (1994) Factors affecting course and survival in Alzheimer's disease. A 9-year longitudinal study. Arch Neurol 51,1213-9. 
[17] Gronning H, Rahmani A, Gyllenborg J, Dessau RB, Hogh P (2012) Does Alzheimer's disease with early onset progress faster than with late onset? A casecontrol study of clinical progression and cerebrospinal fluid biomarkers. Dement Geriatr Cogn Disord 33, 111-7.

[18] Haupt M, Pollmann S, Kurz A (1993) Symptom progression in Alzheimer's disease: relation to onset age and familial aggregation. Results of a longitudinal study. Acta Neurol Scand 88, 349-53.

[19] Katzman R, Brown T, Thal LJ, Fuld PA, Aronson M, Butters N, Klauber M, Wielderholt W, Pay M, Renbing X, Lock W, Hofstetter R, Terry R (1988) Comparison of rate of annual change of mental status score in four independent studies of patients with Alzheimer's disease. Ann Neurol 24, 384-9.

[20] Ortof E, Crystal HA (1989) Rate of progression of Alzheimer's disease. J Am Geriatr Soc 37, 511-4.

[21] Prince M, Knapp M, Guerchet M, McCrone P, Prina M, Comas-Herrera A, Wittenberg R, Adelaja B, Hu B, King D, Rehill A, Salimkumar D (2014) Alzhimers Society Dementia UK: Update, https://www.alzheimers.org.uk/sites/default/files/migrate/downloads/dementia_uk_up date.pdf, published November 2014, Accessed 23 July 2018.

[22] McKhann G, Drachman D, Folstein M, Katzman R, Price D, Stadlan EM (1984) Clinical diagnosis of Alzheimer's disease: report of the NINCDS-ADRDA Work Group under the auspices of Department of Health and Human Services Task Force on Alzheimer's Disease. Neurology 34, 939-44.

[23] Folstein MF, Folstein SE, McHugh PR (1975) "Mini-mental state". A practical method for grading the cognitive state of patients for the clinician. J Psychiatr Res 12, 189-98. 
[24] Hachinski VC, Iliff LD, Zilhka E, Du Boulay GH, McAllister VL, Marshall J, Russell R, Symon L (1975) “Cerebral blood flow in dementia” Arch Neurol 32, 632-7. [25] Singer JD, Willett JB (2003) Applied longitudinal data analysis : modeling change and event occurrence, Oxford University Press, New York, pp 644 [26] Sona A, Ellis KA, Ames D (2013) Rapid cognitive decline in Alzheimer's disease: a literature review. Int Rev Psychiatry 25, 650-8.

[27] Schneider LS, Kennedy RE, Wang G, Cutter GR (2015) Differences in Alzheimer disease clinical trial outcomes based on age of the participants. Neurology 84, $1121-7$.

[28] Spires-Jones TL, Attems J, Thal DR (2017) Interactions of pathological proteins in neurodegenerative diseases. Acta Neuropathol 134,187-205.

[29] Attems J (2017) The multi-morbid old brain. Acta Neuropathol 134, 169-70. [30] Kapasi A, DeCarli C, Schneider JA (2017) Impact of multiple pathologies on the threshold for clinically overt dementia. Acta Neuropathol 134, 171-86.

[31] Frisoni GB, Testa C, Sabattoli F, Beltramello A, Soininen H, Laakso MP (2005) Structural correlates of early and late onset Alzheimer's disease: voxel based morphometric study. J Neurol Neurosurg Psychiatry 76, 112-4.

[32] Nochlin D, van Belle G, Bird TD, Sumi SM (1993) Comparison of the severity of neuropathologic changes in familial and sporadic Alzheimer's disease. Alzheimer Dis Assoc Disord 7, 212-22.

[33] Boyle PA, Yang J, Yu L, Leurgans SE, Capuano AW, Schneider JA, Wilson R, Bennett D (2017) Varied effects of age-related neuropathologies on the trajectory of late life cognitive decline. Brain 140, 804-12.

[34] Clark CM, Sheppard L, Fillenbaum GG, Galasko D, Morris JC, Koss E, Mohs R, Heyman A (1999) Variability in annual mini-mental state examination score in 
patients with probable Alzheimer disease: A clinical perspective of data from the consortium to establish a registry for Alzheimer's disease. Arch Neurol 56, 857-862. 
Table 1 Baseline demographics and clinical characteristics between individuals with YOAD (<65 yrs), MOAD (65-74 yrs) and LOAD (>75 yrs)

\begin{tabular}{|c|c|c|c|c|}
\hline & $\begin{array}{l}\text { Young- } \\
\text { onset } \\
(n=56)\end{array}$ & $\begin{array}{l}\text { Middle-onset } \\
(n=73)\end{array}$ & $\begin{array}{l}\text { Late-onset } \\
(n=176)\end{array}$ & p Value \\
\hline Sex, (\%) male & 45 & 38 & 45 & .62 \\
\hline $\begin{array}{l}\text { Years in } \\
\text { education, mean } \\
\text { (SD) }\end{array}$ & $11.3(2.1) \mathrm{a}$ & $10.6(1.7) \mathrm{b}$ & $10.4(1.6) a, b$ & $<.01$ \\
\hline $\begin{array}{l}\text { Age at diagnosis } \\
\text { (years), mean } \\
\text { (SD) }\end{array}$ & 59.7 (3.2) & $71.1(2.6)$ & $80.2(3.7)$ & $\mathrm{n} / \mathrm{a}$ \\
\hline HIS & $0.7(1.1) \mathrm{a}$ & $1.0(1.6)$ & $1.0(1.1) \mathrm{a}$ & $<.05$ \\
\hline $\begin{array}{l}\text { First symptom } \\
(\%)\end{array}$ & $\begin{array}{l}\text { Memory }=75 \\
\text { Affective }=23 \\
\text { Language }=2 \\
\text { a,b }\end{array}$ & $\begin{array}{l}\text { Memory=97 } \\
\text { Affective }=3 \\
\text { Language }=0 \\
\text { a }\end{array}$ & $\begin{array}{l}\text { Memory=99 } \\
\text { Affective }=1 \\
\text { Language=0 } \\
\text { b }\end{array}$ & $<.01$ \\
\hline $\begin{array}{l}\text { Length of } \\
\text { symptoms (SD) }\end{array}$ & $31.4(26.0)$ & 37.9 (26.5) & $30.6(23.9)$ & .06 \\
\hline $\begin{array}{l}\text { MMSE at } \\
\text { diagnosis, mean } \\
\text { (SD) }\end{array}$ & $\begin{array}{l}18.4(6.1) \mathrm{a}, \\
\mathrm{b}\end{array}$ & $23.2(3.3) \mathrm{a}$ & 23.0 (3.2) b & $<.01$ \\
\hline $\begin{array}{l}\text { CAMCOG at } \\
\text { diagnosis, mean } \\
\text { (SD) }\end{array}$ & $\begin{array}{l}64.0(18.6) \\
a, b\end{array}$ & $78.8(9.4) \mathrm{a}$ & $76.5(10.0) b$ & $<.01$ \\
\hline
\end{tabular}




\begin{tabular}{|l|l|l|l|l|}
\hline $\begin{array}{l}\text { Number of } \\
\text { follow-ups, mean }\end{array}$ & $4.5(2.6) \mathrm{a}$ & $6.0(3.3) \mathrm{a}$ & $4.8(2.5)$ & $<.01$ \\
(SD) & & & & \\
\hline Length follow-up, & $41.0(4-122)$ & $48.0(12-210) \mathrm{a}$, & $36.5(4-149) \mathrm{b}$ & $<.05$ \\
median (range) in & $\mathrm{a}$ & $\mathrm{b}$ & & \\
\hline months & & & & \\
\hline
\end{tabular}

Pairs of letters $a, b, c$ indicate pairs that differ at $p<.05 .3$-way continuous tests are Kruskal-Wallis, 2-way continuous tests are Mann-Whitney. Sex variable $p$ value from Chi-Square test. First symptom variable $p$ value from Fisher's exact test. 
Table 2 Clinical characteristics (percent yes)

\begin{tabular}{|c|c|c|c|c|}
\hline & $\begin{array}{l}\text { Young-onset } \\
(n=56)\end{array}$ & $\begin{array}{l}\text { Middle-onset } \\
(n=73)\end{array}$ & $\begin{array}{l}\text { Late-onset } \\
(n=176)\end{array}$ & P Value \\
\hline Hypertension & $20 a, b$ & $51 a$ & $51 \mathrm{~b}$ & $<.01$ \\
\hline Diabetes & 9 & 14 & 8 & .37 \\
\hline $\begin{array}{l}\text { Hypercholesterolemi } \\
\text { a }\end{array}$ & 18 & 30 & 31 & .15 \\
\hline History MI & 4 & 10 & 10 & .30 \\
\hline Smoker & 39 & 40 & 42 & .83 \\
\hline Alcohol & 70 & 67 & 63 & .73 \\
\hline ACHEI & 93 & 88 & 90 & .42 \\
\hline Memantine & $9 \mathrm{a}$ & $7 \mathrm{~b}$ & $1 \mathrm{a}, \mathrm{b}$ & $<.01$ \\
\hline
\end{tabular}

Pairs of letters $a$ and $b$ indicate pairs that differ at $p<0.05$. All comparisons are Chi Square. 
Figure 1: Study flowchart

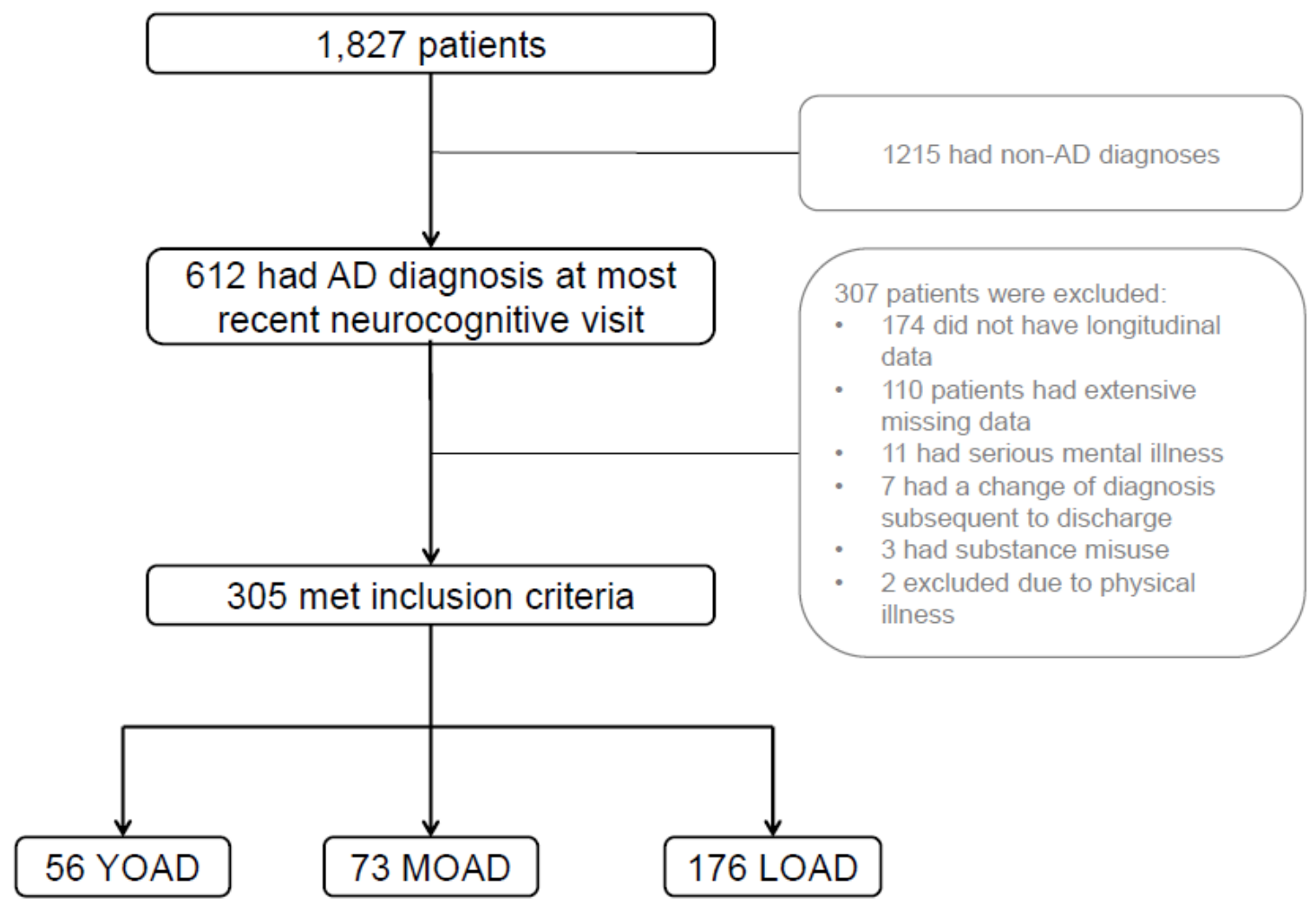


Figure 2. Initial MMSE status (top) and rates of decline (bottom) for YOAD, MOAD and LOAD.
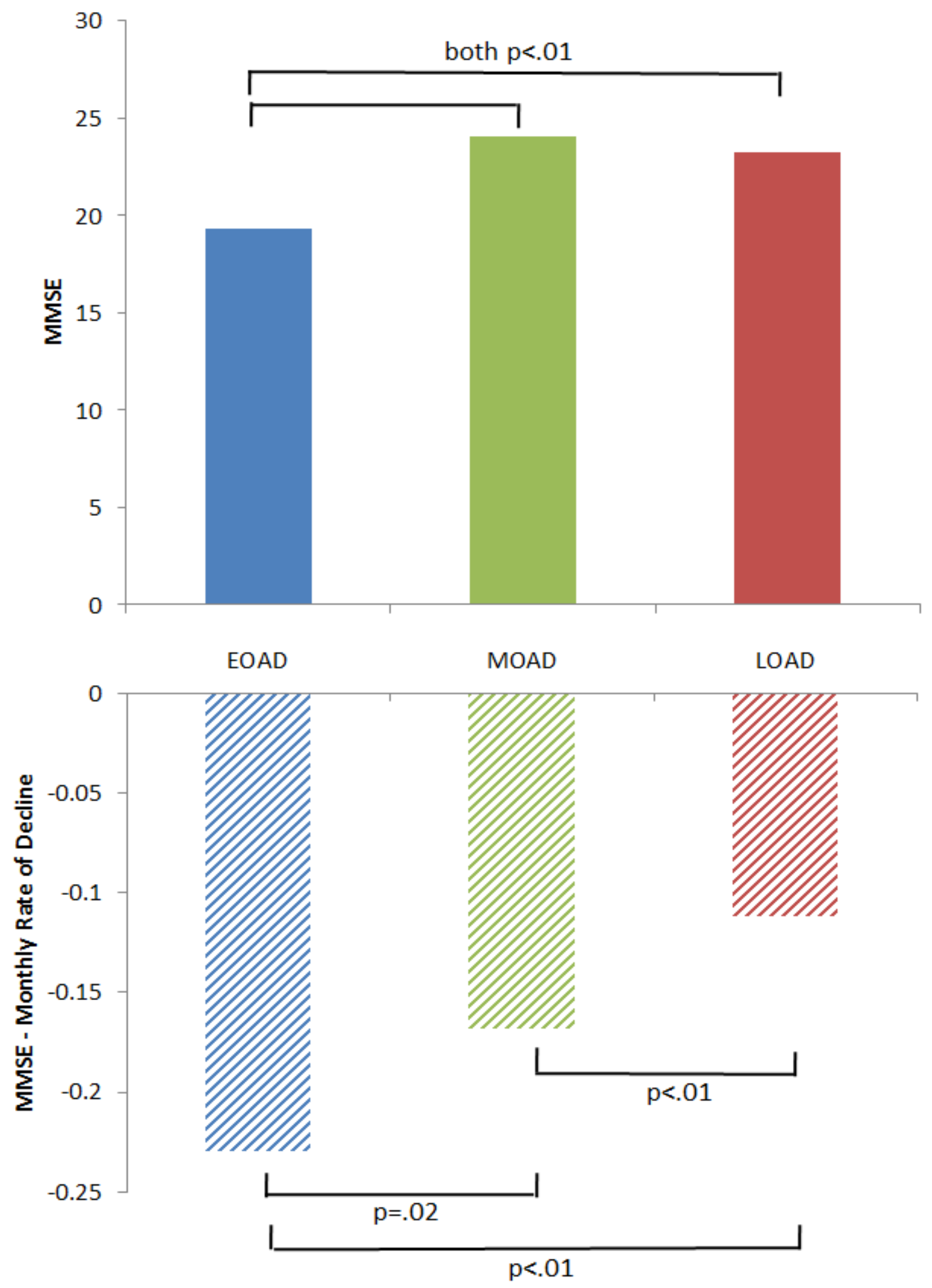